Chakrabarti, A.; Mandal, N.

\section{Solutions of Some Dual Integral Equations}

MSC (1991): 45F10, 33C10

For certain dual integral equations involving trigonometric functions and the Bessel function of zeroth order as their kernels solution methods are described. These methods exploit the fact that, under certain circumstances of practical importance, one of the integrals of each set of the dual integral equations under consideration possesses a "square-root" singularity at the "turning point", i.e., at the point where the boundary conditions change abruptly. The ultimate solutions of the dual integral equations are derived by using the well-known inversion formula for some Abel type integral equations.

\section{Introduction}

Dual integral equations arise, in a natural way (see SNEDDon [5], p. 80, Sneddon and Lowengrub [6], p. 72, and others), while solving certain mixed boundary value problems of mathematical physics in two dimensional as well as in three dimensional axisymmetric cases. Several authors (see Titchmarsh [7], BusBRIdGe [1], Noble [4], Sneddon [5], p. 80, and others) have described varieties of methods to solve dual integral equations.

In the present paper, we have analysed three different sets of dual integral equations for their solutions by utilizing the appropriate singularity behaviour of one of the integrals of each set at the "turning points", where by the term "turning point" we un- derstand the point where the boundary conditions change abruptly, and this will become clear as we proceed further in our analysis. An utilization of such singular behaviour of one of the integrals of a set of dual integral equations has been extensively used by DAvis [2] in a class of mixed boundary value problems arising in viscous fluid flow theory. The first two of the sets of dual integral equations considered in this paper involve the two basic trigonometric functions, i.e., the "sine" and "cosine" function as their kernels, and the third set involves the Bessel function of order zero. As is well-known (see SNEDDON [5], p. 106), dual integral equations involving trigonometric functions as their kernels, occur in the study of a large number of two-dimensional mixed boundary value problems of mathematical physics, and the equations with the Bessel functions as their kernels arise in the study of certain three dimensional axi-symmetric problems, having a cylindrical symmetry.

All the sets of dual integral equations of our concern here have been reduced to Abel type integral equations in each case, and the final solutions are determined by using the standard Abel inversion formula (see SNEDDON [5], p. 41).

Whilst most of the analysis employed in the present paper exists in some form or the other in the literature, the motive behind taking up the present study is to unify the idea of utilizing the singular behaviour of one of the integrals of each set of the dual integral equations in a rather natural and quick manner, reducing the problems to those of solving certain Abel type integral equations. The known results are recovered in the case of each of these sets of dual integral equations considered here.

\section{Some useful results}

The following results (see GRADShtEyn and Ryzhik [3], pp. 419, $681,682,683,730,731)$ are utilized in our analysis $\left(J_{v}(x)\right.$ represents the Bessel function of first kind and of order $v)$ :

$\int_{0}^{\infty} \cos x u J_{0}(u t) \mathrm{d} u= \begin{cases}\frac{1}{\sqrt{t^{2}-x^{2}}}, & x<t \\ 0, & x>t,\end{cases}$
$\int_{0}^{\infty} \sin x u J_{0}(u t) \mathrm{d} u= \begin{cases}0, & x<t \\ \frac{1}{\sqrt{x^{2}-t^{2}}}, & x>t,\end{cases}$

$\int_{0}^{\infty} \cos x u J_{1}(u t) \mathrm{d} u= \begin{cases}\frac{1}{t}, & x<t \\ \frac{-t}{\sqrt{\left(x^{2}-t^{2}\right)}\left(x+\sqrt{x^{2}-t^{2}}\right)}, & x>t,\end{cases}$

$\int_{0}^{1} u J_{0}(x u) \mathrm{d} u=\frac{J_{1}(x)}{x}$

$\int_{t}^{\infty} \frac{u J_{0}(x u) \mathrm{d} u}{\sqrt{x^{2}-t^{2}}}=\frac{\cos x t}{x}$

$\int_{0}^{t} \frac{J_{1}(x u) \mathrm{d} u}{\sqrt{t^{2}-u^{2}}}=\frac{2 \sin ^{2}(u t / 2)}{u t}$,

$\int_{t}^{\infty} \frac{J_{1}(x u) \mathrm{d} u}{\sqrt{u^{2}-t^{2}}}=\frac{\sin x t}{x t}$,

$\int_{0}^{t} \frac{\cos x u \mathrm{~d} u}{\sqrt{t^{2}-u^{2}}}=\frac{\pi}{2} J_{0}(x t)$,

$\int_{0}^{t} \frac{u \sin x u \mathrm{~d} u}{\sqrt{t^{2}-u^{2}}}=\frac{\pi}{2} t J_{1}(x t)$, 
and, finally (see URSELL [8]),

$$
\begin{aligned}
\frac{\pi}{2} J_{1}(a \xi)= & \int_{0}^{a} \frac{\cos \xi u \mathrm{~d} u}{a} \\
& -\frac{1}{a} \int_{a}^{\infty}\left(\frac{u}{\sqrt{u^{2}-a^{2}}}-1\right) \cos \xi u \mathrm{~d} u, \quad a>0 .
\end{aligned}
$$

\section{The detailed analysis}

\subsection{Dual integral equations involving the cosine function kernel}

(I) We first analyse the pair of integral equations given by

$$
\begin{array}{ll}
\int_{0}^{\infty} \frac{A(\xi)}{\xi} \cos x \xi \mathrm{d} \xi=f(x), & 0<x<1, \\
\int_{0}^{\infty} A(\xi) \cos x \xi \mathrm{d} \xi=0, & 1<x<\infty
\end{array}
$$

where $f(x)$ is known, and $A(\xi)$ is to be determined.

To obtain the solution of the pair of equations (1.1) and (1.2), keeping in mind the singularity behaviour of the integral in (1.2) as $x \rightarrow 1-$, we set

$$
\int_{0}^{\infty} A(\xi) \cos x \xi \mathrm{d} \xi=\frac{\mathrm{d}}{\mathrm{d} x}\left[x \int_{x}^{1} \frac{\psi(t) \mathrm{d} t}{\sqrt{t^{2}-x^{2}}}\right], \quad 0<x<1,
$$

where $\psi(t)$ is an unknown differentiable function to be determined, such that $\psi(1) \neq 0$.

The transformation $t=x u$ in the integral followed by differentiation applied to the right hand side of the relation (1.3) shows that it can be expressed as

$$
-\frac{\psi(1)}{\sqrt{1-x^{2}}}+\int_{x}^{1} \frac{\left\{\psi(t)+t \psi^{\prime}(t)\right\} \mathrm{d} t}{\sqrt{t^{2}-x^{2}}},
$$

and this exhibits the singularity structure clearly.

Using the Fourier cosine inversion theorem and the result (R9), from the relations (1.2) and (1.3), we obtain

$$
A(\xi)=\xi \int_{0}^{1} t J_{1}(\xi t) \psi(t) \mathrm{d} t
$$

Substituting from the relation (1.4) into the equations (1.1), and by using the result (R3) along with the Abel inversion formula (cf. [5], p. 41), the unknown function $\psi(t)$ is obtained in the form

$\psi(t)=-\frac{2}{\pi} \frac{\mathrm{d}}{\mathrm{d} t} \int_{0}^{t} \frac{f(u) \mathrm{d} u}{\sqrt{t^{2}-u^{2}}}, \quad 0<t<1$.

Therefore, the equations (1.4) and (1.5) produce the solution of the pair of integral equation (1.1) and (1.2) which is given by

$A(\xi)=\frac{2 \xi^{2}}{\pi} \int_{0}^{1} t J_{0}(\xi t) \mathrm{d} t \int_{0}^{t} \frac{f(u) \mathrm{d} u}{\sqrt{t^{2}-u^{2}}}-\frac{2 \xi J_{1}(\xi)}{\pi} \int_{0}^{1} \frac{f(u) \mathrm{d} u}{\sqrt{1-u^{2}}}$.

This solution (1.6) completely agrees with the one obtained by BUSBRIDGE [1].

(II) The second pair of integral equations of our consideration is

$$
\begin{array}{ll}
\int_{0}^{\infty} A(\xi) \cos x \xi \mathrm{d} \xi=f(x), & 0<x<1, \\
\int_{0}^{\infty} \frac{A(\xi)}{\xi} \cos x \xi \mathrm{d} \xi=0, & 1<x<\infty .
\end{array}
$$

The solution of this pair can be obtained by setting $\int_{0}^{\infty} \frac{A(\xi)}{\xi} \cos x \xi \mathrm{d} \xi=\int_{x}^{1} \frac{\psi(t) \mathrm{d} t}{\sqrt{t^{2}-x^{2}}}, \quad 0<x<1$,

where $\psi(t)$ is a new unknown function to be determined. We emphasize here that the substitution (1.9) takes care of the required behaviour of the integral on the left of the relation (1.8) at the point $x=1$, where it is expected to be $O\left((1-x)^{1 / 2}\right)$, as $x \rightarrow 1-$.

Utilizing the Fourier cosine inversion theorem [7] and the result (R8), the equations (1.8) and (1.9) give

$A(\xi)=\xi \int_{0}^{1} J_{0}(\xi t) \psi(t) \mathrm{d} t$.

Substituting the value of $A(\xi)$ from the relation (1.10) into the equation (1.7), and using the result (R2) along with the Abel inversion formula, we obtain

$\psi(t)=\frac{2 t}{\pi} \int_{0}^{t} \frac{f(u) \mathrm{d} u}{\sqrt{t^{2}-u^{2}}}, \quad 0<t<1$.

Thus, the equations (1.10) and (1.11) give the solution of the dual integral equations (1.7) and (1.8) as

$A(\xi)=\frac{2 \xi}{\pi} \int_{0}^{1} t J_{0}(\xi t) \mathrm{d} t \int_{0}^{t} \frac{f(u) \mathrm{d} u}{\sqrt{t^{2}-u^{2}}}$.

As a special case, (1.12) agrees with Titchmarsh (cf. [7], p. 339).

\subsection{Dual integral equations involving the sine function kernel}

(I) At first, we analyse the dual integral equations

$\begin{array}{ll}\int_{0}^{\infty} \frac{A(\xi)}{\xi} \sin x \xi \mathrm{d} \xi=f(x), & 0<x<1, \\ \int_{0}^{\infty} A(\xi) \sin x \xi \mathrm{d} \xi=0, & 1<x<\infty .\end{array}$

To obtain the solution of the dual integral equations (2.1) and (2.2), we set

$\int_{0}^{\infty} A(\xi) \sin x \xi \mathrm{d} \xi=\frac{\mathrm{d}}{\mathrm{d} x} \int_{x}^{1} \frac{\psi(t) \mathrm{d} t}{\sqrt{t^{2}-x^{2}}}, \quad 0<x<1$,

where $\psi(t)$ is another unknown differentiable function to be determined, such that $\psi(1) \neq 0$.

By using the Fourier sine inversion theorem and the result (R8), from the relations (2.2) and (2.3), we obtain

$A(\xi)=-\xi \int_{0}^{1} J_{0}(\xi t) \psi(t) \mathrm{d} t$.

Now, using the result (R2) and Abel's inversion formula, from the relations (2.1) and (2.4), the unknown function can be obtained as

$\psi(t)=-\frac{2}{\pi} \frac{\mathrm{d}}{\mathrm{d} t} \int_{0}^{t} \frac{u f(u) \mathrm{d} u}{\sqrt{t^{2}-u^{2}}}=-\frac{2 t}{\pi} \int_{0}^{t} \frac{f^{\prime}(u) \mathrm{d} u}{\sqrt{t^{2}-u^{2}}}, \quad 0<t<1$,

where $f(0)=0$ has been used (which is apparent if we make $x \rightarrow 0+$ on both sides of (2.1)). Therefore, from the relations (2.4) and (2.5), the solution of the pair of integral equations (2.1) and (2.2) is given by

$A(\xi)=\frac{2 \xi}{\pi} \int_{0}^{1} t J_{0}(\xi t) \mathrm{d} t \int_{0}^{t} \frac{f^{\prime}(u) \mathrm{d} u}{\sqrt{t^{2}-u^{2}}}$. 
This agrees with the solution of one of the special cases of the dual integral equations handled by BusBridge [1]

(II) Secondly, we consider the dual integral equations

$$
\begin{array}{ll}
\int_{0}^{\infty} A(\xi) \sin x \xi \mathrm{d} \xi=f(x), & 0<x<1, \\
\int_{0}^{\infty} \frac{A(\xi)}{\xi} \sin x \xi \mathrm{d} \xi=0, & 1<x<\infty .
\end{array}
$$

The solution of this pair can be obtained by setting

$\int_{0}^{\infty} \frac{A(\xi)}{\xi} \sin x \xi \mathrm{d} \xi=x \int_{x}^{1} \frac{\psi(t) \mathrm{d} t}{\sqrt{t^{2}-x^{2}}}, \quad 0<x<1$,

where $\psi(t)$ is an unknown differentiable function to be determined.

Using the Fourier sine inversion theorem [7] and the result (R9), the equations (2.8) and (2.9) give rise to the following expression for the function $A(\xi)$ :

$A(\xi)=\xi \int_{0}^{1} t J_{1}(\xi t) \psi(t) \mathrm{d} t$.

Inserting relation (2.10) into equation (2.7), we find

$\frac{\mathrm{d}}{\mathrm{d} x} \int_{0}^{1} t \psi(t)\left(\int_{0}^{\infty} J_{1}(\xi t) \cos x \xi \mathrm{d} \xi\right) \mathrm{d} t=-f(x), \quad 0<x<1$.

Now, using the result (R3), and integrating the equation (2.11) on both sides, we find

$\int_{0}^{x} \frac{\psi(t) \mathrm{d} t}{\sqrt{t^{2}-x^{2}}}=\frac{1}{x} \int_{0}^{x} f(u) \mathrm{d} u, \quad 0<x<1$.

The equation (2.12) is an Abel integral equation, and, therefore, applying Abel's inversion formula, we obtain

$\psi(t)=-\frac{2}{\pi t} \int_{0}^{t} \frac{u f(u) \mathrm{d} u}{\sqrt{t^{2}-u^{2}}}, \quad 0<t<1$.

Thus, the solution of the dual integral equations (2.7) and (2.8) can be obtained from the relations (2.10) and (2.13), and we find

$A(\xi)=\frac{2 \xi}{\pi} \int_{0}^{1} J_{1}(\xi t) \mathrm{d} t \int_{0}^{t} \frac{u f(u) \mathrm{d} u}{\sqrt{t^{2}-u^{2}}}$.

This agrees with one of the special cases of the solution obtained by NOBLE [4].

3.3 Dual integral equations involving the Bessel function kernel

(I) In this Section, we analyse the following dual integral equations:

$$
\begin{array}{ll}
\int_{0}^{\infty} \xi A(\xi) J_{0}(x \xi) \mathrm{d} \xi=\frac{\pi}{2}, & 0<x<1, \\
\int_{0}^{\infty} A(\xi) J_{0}(x \xi) \mathrm{d} \xi=0, & 1<x<\infty
\end{array}
$$

where $A(\xi)$ is the unknown function to be determined.

To obtain the solution of (3.1) and (3.2), we set

$$
\int_{0}^{\infty} \xi A(\xi) J_{0}(x \xi) \mathrm{d} \xi=\frac{\pi}{2 x} \frac{\mathrm{d}}{\mathrm{d} x} \int_{1}^{x} \frac{t \psi(t) \mathrm{d} t}{\sqrt{x^{2}-t^{2}}}, \quad 0<x<\infty,
$$

where $\psi(t)$ is another unknown differentiable function to be determined, such that $\psi(1) \neq 0$, and this exhibits the appropriate singularity behaviour of the integral on the left at the turning point $x=1$.

Utilizing the Hankel inversion theorem ([5], p. 29) along with the results (R4) and (R7), from the relations (3.1) and (3.3), we obtain

$A(\xi)=\frac{\pi}{2}\left[\frac{J_{1}(\xi)}{\xi}+\int_{1}^{\infty} \psi(t) \sin \xi t \mathrm{~d} t\right]$.

Substituting the expression (3.4) into equation (3.2), and then using the result (R2), we obtain an Abel type intergral equation as given by

$\int_{x}^{\infty} \frac{\psi(t) \mathrm{d} t}{\sqrt{t^{2}-x^{2}}}=-\int_{0}^{\infty} \frac{J_{0}(\xi x) J_{1}(\xi)}{\xi} \mathrm{d} \xi, \quad 1<x<\infty$.

Using Abel's inversion formula, and then interchanging the orders of integration, the unknown function $\psi(t)$ can be obtained in the form

$\psi(t)=\frac{2}{\pi} \int_{0}^{\infty} \frac{J_{1}(\xi)}{\xi}\left(\frac{\mathrm{d}}{\mathrm{d} t} \int_{t}^{\infty} \frac{x J_{0}(\xi x)}{\sqrt{x^{2}-t^{2}}} \mathrm{~d} x\right) \mathrm{d} \xi, \quad 1<t<\infty$.

Then, using the result (R5) for the above relation, we find that

$\psi(t)=-\frac{2}{\pi\left(t+\sqrt{t^{2}-1}\right)}, \quad 1<t<\infty$.

Substituting this into the equation (3.4), and simplifying with the help of the result (R10), the solution of the dual integral equations can be obtained as given by

$A(\xi)=\frac{\sin \xi}{\xi^{2}}-\frac{\cos \xi}{\xi}=-\frac{\mathrm{d}}{\mathrm{d} \xi}\left(\frac{\sin \xi}{\xi}\right)$,

and this agrees with the one cited in DAVIS' paper [2] as well as in SNEDDON's book [4], p. 77.

(II) At last we consider the pair of integral equations

$\int_{0}^{\infty} A(\xi) J_{0}(x \xi) \mathrm{d} \xi=\frac{\pi}{2}, \quad 0<x<1$,

$\int_{0}^{\infty} \xi A(\xi) J_{0}(x \xi) \mathrm{d} \xi=0, \quad 1<x<\infty$.

For the solution of this pair of integral equations, we set

$\int_{0}^{\infty} \xi A(\xi) J_{0}(x \xi) \mathrm{d} \xi=\frac{1}{x} \frac{\mathrm{d}}{\mathrm{d} x} \int_{x}^{1} \frac{t \psi(t) \mathrm{d} t}{\sqrt{t^{2}-x^{2}}}, \quad 0<x<1$,

where $\psi(t)$ is a new unknown differentiable function with $\psi(1) \neq 0$ to be determined.

By the Hankel inversion theorem [5], p. 29, and use of the result (R6), from the relations (3.9) and (3.10), the solution of the above dual integral equations is obtained, in terms of the unknown function $\psi(t)$, as

$A(\xi)=-\int_{0}^{1} \psi(t) \cos \xi t \mathrm{~d} t$.

Using this for equation (3.8) along with the result (R1), an Abel type integral equation is obtained as

$\int_{0}^{x} \frac{\psi(t) \mathrm{d} t}{\sqrt{x^{2}-t^{2}}}=-\frac{\pi}{2}, \quad 0<x<1$,

the solution of which is given by

$\psi(t)=-1, \quad 0<t<1$. 
Therefore, from the relations (3.11) and (3.13), the solution of the dual integral equations (3.8) and (3.9) is obtained in the form

$A(\xi)=\frac{\sin \xi}{\xi}$,

which agrees with the corresponding result available in SNEDDON's book [5], p. 64 .

Acknowledgement: The second author is grateful to IIScTIFR centre for his short-term visit to Department of Mathematics, IISc, Bangalore, and to CSIR, New Delhi, for the award of a research Associateship. The authors also thank the referees for their suggestions to improve the paper.

\section{References}

1 Busbridge, I. W.: Dual integral equations. Proc. London Math. Soc. 44 (1938), $115-129$.

2 Davis, A. M. J.: A translating disk in a Sampson flow; pressure driven flow through concentric holes in parallel walls. Quart. J. Mech. Appl. Math. 44 (1991) 3, $471-486$.
3 Gradshteyn, I. S.; Ryzhik, I. M.: Tables of integrals, series and products. Academic Press 1992

4 Noble, B.: The solution of Bessel function dual integral equations by a multiplyingfactor method. Proc. Cambridge Philos. Soc. 59 (1963), 351-362.

5 Sneddon, I. N.: Mixed boundary value problems in potential theory. North-Holland Publishing, Amsterdam 1966.

6 Sneddon, I. N.; Lowengrub, M.: Crack problems in the mathematical theory of elasticity. John Wiley and Sons, New York 1969

7 Titchmarsh, E. C.: Introduction to theory of Fourier integrals. Clarendon Press, Oxford 1948 (Second Edition).

8 URSELL, F.: The effect of a fixed vertical barrier on surface waves in deep water. Proc. Cambridge Philos. Soc 43 (1947), 374-382.

Addresses: Prof. Dr. Aloknath Chakrabarti, Dept. of Mathematics, Indian Institute of Science, Bangalore560012, India; Dr. NAnigopal Mandal, Physics and Applied Mathematics Unit, Indian Statistical Institute, 203 B.T.Road, Calcutta-700 035, India 